\title{
EIGENFUNCTION EXPANSIONS OF ULTRADIFFERENTIABLE FUNCTIONS AND ULTRADISTRIBUTIONS. II. TENSOR REPRESENTATIONS
}

\author{
APARAJITA DASGUPTA AND MICHAEL RUZHANSKY
}

\begin{abstract}
In this paper we analyse the structure of the spaces of coefficients of eigenfunction expansions of functions in Komatsu classes on compact manifolds, continuing the research in our paper [Trans. Amer. Math. Soc. 368 (2016), pp.8481-8498]. We prove that such spaces of Fourier coefficients are perfect sequence spaces. As a consequence we describe the tensor structure of sequential mappings on spaces of Fourier coefficients and characterise their adjoint mappings. In particular, the considered classes include spaces of analytic and Gevrey functions, as well as spaces of ultradistributions, yielding tensor representations for linear mappings between these spaces on compact manifolds.
\end{abstract}

\section{Contents}

1. Introduction

2. Fourier analysis on compact manifolds

3. Sequence spaces and sequential linear mappings

4. Tensor representations for Komatsu classes and their $\alpha$-duals

5. Beurling class of ultradifferentiable functions and ultradistributions References

\section{INTRODUCTION}

The present paper is a continuation of our paper [4] where we have characterised Komatsu spaces of ultradifferentiable functions and ultradistributions on compact manifolds in terms of the eigenfunction expansions related to positive elliptic operators. In particular, these classes include the spaces of analytic, Gevrey, and smooth functions as well as the corresponding dual spaces of distributions and ultradistributions, in both Roumieu and Beurling settings.

In particular, this extended the earlier characterisations of analytic functions on compact manifolds in terms of the eigenfunction expansions by Seeley 23] (see also [22]), and characterisations of Gevrey spaces and ultradistributions on tori [24] and on compact Lie groups and homogeneous spaces [3].

Received by the editors July 7, 2017.

2010 Mathematics Subject Classification. Primary 46F05; Secondary 58J40, 22E30.

Key words and phrases. Gevrey spaces, ultradistributions, Komatsu classes, compact manifolds.

The second author was supported by the EPSRC Grants EP/K039407/1 and EP/R003025/1, and by the Leverhulme Research Grant RPG-2017-151. No new data was collected or generated during the course of this research. 
For example, if $E$ is a positive elliptic pseudo-differential operator on a compact manifold $X$ without boundary and $\lambda_{j}$ denotes its eigenvalues in the ascending order, then smooth functions on $X$ can be characterised in terms of their Fourier coefficients:

$$
f \in C^{\infty}(X) \Longleftrightarrow \forall N \exists C_{N}:|\widehat{f}(j, k)| \leq C_{N} \lambda_{j}^{-N} \text { for all } j \geq 1,1 \leq k \leq d_{j},
$$

where $\widehat{f}(j, k)=\left(f, e_{j}^{k}\right)_{L^{2}}$ with $e_{j}^{k}$ being the $k^{\text {th }}$ eigenfunction corresponding to the eigenvalue $\lambda_{j}$ (of multiplicity $d_{j}$ ); see (2.1). If $X$ and $E$ are analytic, the result of Seeley 23 can be reformulated as

$$
f \text { is analytic } \Longleftrightarrow \exists L>0 \exists C:|\widehat{f}(j, k)| \leq C e^{-L \lambda_{j}^{1 / \nu}} \text { for all } j \geq 1,1 \leq k \leq d_{j},
$$

where $\nu$ is the order of the pseudo-differential operator $E$. In [4] we extended such characterisations to Gevrey classes and, more generally, to Komatsu classes of ultradifferentiable functions and the corresponding classes of ultradistributions.

In this paper we continue this analysis showing that the appearing spaces of coefficients with respect to expansions in eigenfunctions of positive elliptic operators are perfect spaces in the sense of the theory of sequence spaces (see, e.g., Köthe [10]). Consequently, we obtain tensor representations for linear mappings between spaces of ultradifferentiable functions and the corresponding spaces of ultradistributions. Such discrete representations in a given basis are useful in different areas of timefrequency analysis, in partial differential equations, and in numerical investigations. Due to possible multiplicities of eigenvalues the mappings beget the tensor structure rather than the matrix one as it would be in the case of simple eigenvalues, and our results are new for both situations.

Our analysis is based on the global Fourier analysis on compact manifolds which was consistently developed in [5], with a number of subsequent applications, for example to the spectral properties of operators [7, or to the wave equations for the Landau Hamiltonian [20. The corresponding version of the Fourier analysis based on expansions with respect to biorthogonal systems of eigenfunctions of nonself-adjoint operators has been developed in [19], with a subsequent extension in [21.

The obtained characterisations of Komatsu classes found their applications, for example for the well-posedness problems for weakly hyperbolic partial differential equations 8 . The spaces of coefficients of eigenfunction expansions in $\mathbb{R}^{n}$ with respect to the eigenfunctions of the harmonic oscillator have been analysed in [9], and the corresponding Komatsu classes have been investigated in [26]. The original Komatsu spaces of ultradifferentiable functions and ultradistributions have appeared in the works [11 13] by Komatsu (see also Rudin [18), extending the original works by Roumieu [17]. The universality of the spaces of Gevrey functions on the torus has been established in [25].

The regularity properties of spaces of distributions and ultradistributions have been analysed in [15, and their convolution properties appeared in [14.

The characterisations in terms of the eigenfunction expansions provide for descriptions alternative to those using the classical Fourier analysis, with applications in the theory of partial differential equations; see, e.g., [16]. For some other applications of this type of analysis one can see, e.g., [1,2].

The paper in organised as follows. In Section 2 we briefly recall the constructions leading to the global Fourier analysis on compact manifolds. In Section 3 we very 
briefly recall the relevant definitions from the theory of sequence spaces. In Section 4 we present the main results of this paper and their proofs. In Section 5 we first recall the definitions for the Beurling version of the spaces and then give the statement of the corresponding adjointness Theorem 4.7 in this case.

In this paper we adopt the notation $\mathbb{N}_{0}=\mathbb{N} \cup\{0\}$.

\section{FOURIER ANALYSIS ON COMPACT MANIFOLDS}

Let $X$ be a closed $C^{\infty}$-manifold of dimension $n$ endowed with a fixed measure $d x$. We first recall an abstract statement from [5, Theorem 2.1] giving rise to the Fourier analysis on $L^{2}(X)$.

Theorem 2.1. Let $\mathcal{H}$ be a complex Hilbert space and let $\mathcal{H}^{\infty} \subset \mathcal{H}$ be a dense linear subspace of $\mathcal{H}$. Let $\left\{d_{j}\right\}_{j \in \mathbb{N}_{0}} \subset \mathbb{N}$ and let $\left\{e_{j}^{k}\right\}_{j \in \mathbb{N}_{0}, 1 \leq k \leq d_{j}}$ be an orthonormal basis of $\mathcal{H}$ such that $e_{j}^{k} \in \mathcal{H}^{\infty}$ for all $j$ and $k$. Let $H_{j}:=\operatorname{span}\left\{e_{j}^{k}\right\}_{k=1}^{d_{j}}$, and let $P_{j}: \mathcal{H} \rightarrow H_{j}$ be the orthogonal projection. For $f \in \mathcal{H}$, we denote $\widehat{f}(j, k):=\left(f, e_{j}^{k}\right)_{\mathcal{H}}$ and let $\widehat{f}(j) \in \mathbb{C}^{d_{j}}$ denote the column of $\widehat{f}(j, k), 1 \leq k \leq d_{j}$. Let $T: \mathcal{H}^{\infty} \rightarrow \mathcal{H}$ be a linear operator. Then the following conditions (i)-(iii) are equivalent:

(i) For each $j \in \mathbb{N}_{0}$, we have $T\left(H_{j}\right) \subset H_{j}$.

(ii) For each $l \in \mathbb{N}_{0}$ there exists a matrix $\sigma(l) \in \mathbb{C}^{d_{l} \times d_{l}}$ such that for all $e_{j}^{k}$,

$$
\widehat{T e_{j}^{k}}(l, m)=\sigma(l)_{m k} \delta_{j l} .
$$

(iii) If in addition all $e_{j}^{k}$ are in the domain of $T^{*}$, then for each $l \in \mathbb{N}_{0}$ there exists a matrix $\sigma(l) \in \mathbb{C}^{d_{l} \times d_{l}}$ such that for all $f \in \mathcal{H}^{\infty}$ we have

$$
\widehat{T f}(l)=\sigma(l) \widehat{f}(l) .
$$

The matrices in (ii) and (iii) coincide.

The equivalent properties (i)-(iii) follow from the condition:

(iv) For each $j \in \mathbb{N}_{0}$, we have $T P_{j}=P_{j} T$ on $\mathcal{H}^{\infty}$.

If, in addition, $T$ extends to a bounded operator $T \in \mathcal{L}(H)$, then (iv) is equivalent to (i)-(iii).

Under the assumptions of Theorem 2.1 we have the direct sum decomposition

$$
\mathcal{H}=\bigoplus_{j=0}^{\infty} H_{j}, \quad H_{j}=\operatorname{span}\left\{e_{j}^{k}\right\}_{k=1}^{d_{j}},
$$

and we have $d_{j}=\operatorname{dim} H_{j}$. Here we will consider $\mathcal{H}=L^{2}(X)$ for a compact manifold $X$ with $H_{j}$ being the eigenspaces of an elliptic positive pseudo-differential operator E.

The eigenvalues of $E$ (counted without multiplicities) form a sequence $\lambda_{j}, j \in \mathbb{N}$, which we order so that

$$
0=: \lambda_{0}<\lambda_{1}<\lambda_{2}<\ldots
$$

For each eigenvalue $\lambda_{j}$, there is the corresponding finite dimensional eigenspace $H_{j}$ of functions on $X$, which are smooth due to the ellipticity of $E$. We set

$$
d_{0}:=\operatorname{dim} H_{0}, \quad H_{0}:=\operatorname{ker} E, \quad \lambda_{0}:=0 .
$$

Since the operator $E$ is elliptic, it is Fredholm, hence also $d_{0}<\infty$. 
We denote by $\Psi_{+e}^{\nu}(X)$ the space of positive elliptic pseudo-differential operators on order $\nu>0$ on $M$. Here we recall a useful relation between the sequences $\lambda_{j}$ and $d_{j}$ of eigenvalues of $E \in \Psi_{+e}^{\nu}(X)$ and their multiplicities from [5].

Proposition 2.2. Let $X$ be a closed manifold of dimension n, and let $E \in \Psi_{+e}^{\nu}(X)$, with $\nu>0$. Then there exists a constant $C>0$ such that we have

$$
d_{j} \leq C\left(1+\lambda_{j}\right)^{\frac{n}{\nu}}
$$

for all $j \geq 1$. Moreover, we also have

$$
\sum_{j=1}^{\infty} d_{j}\left(1+\lambda_{j}\right)^{-q}<\infty \text { if and only if } q>\frac{n}{\nu} .
$$

For $f \in L^{2}(X)$, by definition we have the Fourier series decomposition

$$
f=\sum_{j=0}^{\infty} \sum_{k=1}^{d_{j}} \widehat{f}(j, k) e_{j}^{k}
$$

The Fourier coefficients of $f \in L^{2}(X)$ with respect to the orthonormal basis $\left\{e_{j}^{k}\right\}$ are denoted by

$$
\mathcal{F} f(j, k)=\widehat{f}(j, k):=\left(f, e_{j}^{k}\right)_{L^{2}} .
$$

We denote the space of Fourier coefficients by

$$
\Sigma=\left\{v=\left(v_{l}\right)_{l \in \mathbb{N}_{0}}, v_{l} \in \mathbb{C}^{d_{l}}\right\} .
$$

Since $\left\{e_{j}^{k}\right\}_{j \geq 0}^{1 \leq k \leq d_{j}}$ is a complete orthonormal system of $L^{2}(X)$ we have the Plancherel formula

$$
\|f\|_{L^{2}(X)}^{2}=\left(\sum_{j=0}^{\infty} \sum_{k=1}^{d_{j}}|\widehat{f}(j, k)|^{2}\right)^{1 / 2}=\|\widehat{f}\|_{l^{2}\left(\mathbb{N}_{0}, \Sigma\right)}^{2}=: \sum_{j=0}^{\infty}\|\widehat{f}(j)\|_{\mathrm{HS}}^{2},
$$

where we interpret $\widehat{f}$ as an element of the space

$$
l^{2}\left(\mathbb{N}_{0}, \Sigma\right)=\left\{h: \mathbb{N}_{0} \rightarrow \prod_{j} \mathbb{C}^{d_{j}}: h(j) \in \mathbb{C}^{d_{j}}, \sum_{j=0}^{\infty} \sum_{k=1}^{d_{j}}|h(j, k)|^{2}<\infty\right\} .
$$

We endow $l^{2}\left(\mathbb{N}_{0}, \Sigma\right)$ with the norm

$$
\|h\|_{l^{2}\left(\mathbb{N}_{0}, \Sigma\right)}=\left(\sum_{j=0}^{\infty} \sum_{k=1}^{d_{j}}|h(j, k)|^{2}\right)^{1 / 2} .
$$

We can think of $\mathcal{F}=\mathcal{F}_{X}$ as of the Fourier transform which is an isometry form $L^{2}(X)$ onto $l^{2}\left(\mathbb{N}_{0}, \Sigma\right)$. The inverse of this Fourier transform can be then expressed by

$$
\left(\mathcal{F}^{-1} h\right)(x)=\sum_{j=0}^{\infty} \sum_{k=1}^{d_{j}} h(j, k) e_{j}^{k}(x)
$$


If $f \in L^{2}(X)$ we can write

$$
\widehat{f}(j)=\left(\begin{array}{c}
\widehat{f}(j, 1) \\
\vdots \\
\vdots \\
\vdots \\
\vdots \\
\widehat{f}\left(j, d_{j}\right)
\end{array}\right) \in \mathbb{C}^{d_{j}}
$$

thus thinking of the Fourier transforn always as a column vector. In particular, we think of

$$
\widehat{e}_{j}^{k}(l)=\left(\widehat{e}_{j}^{k}(l, m)\right)_{m=1}^{d_{l}}
$$

as of a column, and we notice that

$$
\widehat{e_{j}^{k}}(l, m)=\delta_{j l} \delta_{k m} .
$$

\section{Sequence SPACES AND SEqUential Linear mapPings}

We briefly recall that a sequence space $E$ is a linear subspace of

$$
\mathbb{C}^{\mathbb{Z}}=\left\{a=\left(a_{j}\right) \mid a_{j} \in \mathbb{C}, j \in \mathbb{Z}\right\} .
$$

The dual $\widehat{E}$ ( $\alpha$-dual in the terminology of G. Köthe [10]) is a sequence space defined by

$$
\widehat{E}=\left\{a \in \mathbb{C}^{\mathbb{Z}}: \sum_{j \in \mathbb{Z}}\left|u_{j}\right||| a_{j} \mid<\infty \text { for all } u \in E\right\}
$$

A sequence space $E$ is called perfect if $\widehat{\widehat{E}}=E$. A sequence space is called normal if $u=\left(u_{j}\right) \in E$ implies $|u|=\left(\left|u_{j}\right|\right) \in E$. A dual space $\widehat{E}$ is normal so that any perfect space is normal.

A pairing $\langle\cdot, \cdot\rangle_{E}$ on $E$ is a bilinear function on $E \times \widehat{E}$ defined by

$$
\langle u, v\rangle_{E}=\sum_{j \in \mathbb{Z}} u_{j} v_{j} \in \mathbb{C},
$$

which converges absolutely by the definition of $\widehat{E}$.

Definition 3.1. $\phi: E \rightarrow \mathbb{C}$ is called a sequential linear functional if there exists some $a \in \widehat{E}$ such that $\phi(u)=\langle u, a\rangle_{E}$ for all $u \in E$. We abuse the notation by also writing $a: E \rightarrow \mathbb{C}$ for this mapping.

Definition 3.2. A mapping $f: E \rightarrow F$ between two sequence spaces is called a sequential linear mapping if

(i) $f$ is algebraically linear,

(ii) for any $v \in \widehat{F}$, the composed mapping $v \circ f \in \widehat{E}$. 


\section{Tensor Representations For Komatsu Classes And their $\alpha$-DUALS}

Let $M_{k}$ be a sequence of positive numbers such that

(M.0) $M_{0}=1$.

(M.1) (Stability) $M_{k+1} \leq A H^{k} M_{k}, k=0,1,2, \ldots$

(M.2) $M_{2 k} \leq A H^{k} \min _{0 \leq q \leq k} M_{q} M_{k-q}, k=0,1,2, \ldots$, for some $A, H>0$.

In a sequence of papers [11 13 Komatsu investigated classes of ultradifferentiable functions on $\mathbb{R}^{n}$ associated to the sequence $M_{k}$, namely, the spaces of functions $\Psi \in C^{\infty}\left(\mathbb{R}^{n}\right)$ such that for every compact $K \subset \mathbb{R}^{n}$ there exist $h>0$ and a constant $C>0$ such that

$$
\sup _{x \in K}\left|\partial^{\alpha} \psi(x)\right| \leq C h^{|\alpha|} M_{|\alpha|}
$$

holds for all multi-indices $\alpha \geq 0$. Similar to the case of usual distributions given a space of ultradifferentiable functions satisfying (4.1) we can define a space of ultradistributions as its dual.

We now recall the analogous definition of the Komatsu ultradifferentiable functions $\Gamma_{\left\{\mathcal{M}_{k}\right\}}(X)$ and its $\alpha$-dual $\left[\Gamma_{\left\{\mathcal{M}_{k}\right\}}(X)\right]^{\wedge}$. Here, as before, $X$ is a compact manifold without boundary and $E \in \Psi_{+e}^{\nu}(X)$ with $\nu>0$.

Definition 4.1. The class $\Gamma_{\left\{M_{k}\right\}}(X)$ is the space of $C^{\infty}$ functions $\phi$ on $X$ such that there exist $h>0$ and $C>0$ such that we have

$$
\left\|E^{k} \phi\right\|_{L^{2}(X)} \leq C h^{\nu k} M_{\nu k}, \quad k=0,1,2, \ldots,
$$

where $\nu \in \mathbb{N}$ is the order of the positive elliptic pseudo-differential operator $E$.

In 4] we have characterised the class $\Gamma_{\left\{M_{k}\right\}}(X)$ in terms of the eigenvalues of the operator $E$. We assume that

(M.3) For some $l, C_{l}>0$ we have $k ! \leq C_{l} l^{k} M_{k}$, for all $k \in \mathbb{N}_{0}$.

In what follows, for $w_{l} \in \mathbb{C}^{d_{l}}$ we write

$$
\left\|w_{l}\right\|_{\text {HS }}:=\left(\sum_{j=1}^{d_{l}}\left|\left(w_{l}\right)_{j}\right|^{2}\right)^{1 / 2} .
$$

Theorem 4.1 (4]). Assume conditions (M.0), (M.1), (M.2), (M.3). Then $\phi \in$ $\Gamma_{\left\{M_{k}\right\}}(X)$ if and only if there exist constants $C>0$ and $L>0$ such that

$$
\|\widehat{\phi}(l)\|_{\mathrm{HS}} \leq C \exp \left\{-M\left(L \lambda_{l}^{1 / \nu}\right)\right\} \quad \text { for all } l \geq 1,
$$

where

$$
M(r):=\sup _{k \in \mathbb{N}_{0}} \log \frac{r^{\nu k}}{M_{\nu k}} .
$$

Example 4.2. As an example, for the (Gevrey-Roumieu) class of ultradifferentiable functions

$$
\gamma^{s}(X)=\Gamma_{\left\{(k !)^{s}\right\}}(X), \quad 1<s<\infty,
$$

we have $M(r) \simeq r^{1 / s}$. This is also true for $s=1$, characterising the class of analytic functions if the manifold is analytic. The class $\gamma^{s}(X)$ coincides with the usual Gevrey class of functions on a manifold $X$ defined in terms of their localisations. 
Based on Theorem 4.1 we can then write

$\Gamma_{\left\{\mathcal{M}_{k}\right\}}(X)=\left\{[\widehat{\phi}(l)]_{l \in \mathbb{N}_{0}}: \phi \in C^{\infty}(X) \exists C>0\right.$ such that

$$
\left.\|\widehat{\phi}(l)\|_{\mathrm{HS}} \leq C \exp \left\{-M\left(L \lambda_{l}^{1 / \nu}\right)\right\} \forall l \geq 1\right\} .
$$

For $\phi \in \Gamma_{\left\{\mathcal{M}_{k}\right\}}(X)$ we will write

$$
\phi \approx[\widehat{\phi}(l)]_{l \in \mathbb{N}_{0}}
$$

so that $\Gamma_{\left\{\mathcal{M}_{k}\right\}}(X)$ can be thought of as a sequence space, but it will be convenient to view it as a subspace of $\Sigma$ defined in (2.2), taking into account the dimensions of the eigenspaces of the operator $E$.

Next we recall the definition of the $\alpha$-dual of the space $\Gamma_{\left\{\mathcal{M}_{k}\right\}}(X)$ (following [4]).

The $\alpha$-dual of the space $\Gamma_{\left\{\mathcal{M}_{k}\right\}}(X)$ of ultradifferentiable functions, denoted by $\left[\Gamma_{\left\{\mathcal{M}_{k}\right\}}(X)\right]^{\wedge}$, is given by

$$
\left\{v=\left(v_{l}\right)_{l \in \mathbb{N}_{0}} \in \Sigma, v_{l} \in \mathbb{C}^{d_{l}}: \sum_{l=0}^{\infty} \sum_{j=1}^{d_{l}}\left|\left(v_{l}\right)_{j} \| \widehat{\phi}(l, j)\right|<\infty \text { for all } \phi \in \Gamma_{\left\{\mathcal{M}_{k}\right\}}(X)\right\} .
$$

We also recall the following characterisations of the $\alpha$-duals established in 4 .

Theorem 4.3. Assume conditions (M.0), (M.1), (M.2), (M.3). The following statements are equivalent:

(i) $v \in\left[\Gamma_{\left\{\mathcal{M}_{k}\right\}}(X)\right]^{\wedge}$;

(ii) for every $L>0$ we have

$$
\sum_{l=0}^{\infty} \exp \left(-M\left(L \lambda_{l}^{1 / \nu}\right)\right)\left\|v_{l}\right\|_{\mathrm{HS}}<\infty ;
$$

(iii) for every $L>0$ there exists $K_{L}>0$ such that

$$
\left\|v_{l}\right\|_{\mathrm{HS}} \leq K_{L} \exp \left(M\left(L \lambda_{l}^{1 / \nu}\right)\right)
$$

holds for all $l \in \mathbb{N}_{0}$.

We will now show that the space $\Gamma_{\left\{\mathcal{M}_{k}\right\}}(X)$ is perfect. In the proof as well as in further proofs the following estimate will be useful:

$$
\left\|e_{l}^{j}\right\|_{L^{\infty}(X)} \leq C \lambda_{l}^{\frac{n-1}{2 \nu}} \text { for all } l \geq 1 .
$$

This estimate follows, for example, from the local Weyl law [6, Theorem 5.1]; see also [5, Lemma 8.5].

Theorem 4.4. Let $X$ be a compact manifold and assume conditions (M.0), (M.1), (M.2), (M.3). Then $\Gamma_{\left\{\mathcal{M}_{k}\right\}}(X)$ is a perfect space, that is, we have

$$
\Gamma_{\left\{\mathcal{M}_{k}\right\}}(X)=\left[\Gamma_{\left\{\mathcal{M}_{k}\right\}}(X)\right]^{\wedge},
$$

where

$$
\begin{aligned}
& {\left[\Gamma_{\left\{\mathcal{M}_{k}\right\}}(X)\right]^{\wedge}} \\
& \quad=\left\{w=\left(w_{l}\right)_{l \in \mathbb{N}_{0}} \in \Sigma: \sum_{l=0}^{\infty} \sum_{j=1}^{d_{l}}\left|\left(w_{l}\right)_{j}\right|\left|\left(v_{l}\right)_{j}\right|<\infty \forall v \in\left[\Gamma_{\left\{\mathcal{M}_{k}\right\}}(X)\right]^{\wedge}\right\} .
\end{aligned}
$$


To prove this we first establish the following lemma:

Lemma 4.5. We have $w \in\left[\widehat{\Gamma_{\left\{\mathcal{M}_{k}\right\}}(X)}\right]^{\wedge}$ if and only if there exists $L>0$ such that

$$
\sum_{l=0}^{\infty} \exp \left(M\left(L \lambda_{l}^{1 / \nu}\right)\right)\left\|w_{l}\right\|_{\mathrm{HS}}<\infty .
$$

Proof of Lemma 4.5, $\Longrightarrow$ : For $L>0$ we consider the echelon space

$$
D_{L}=\left\{v=\left(v_{l}\right) \in \Sigma: \exists C>0:\left|\left(v_{l}\right)_{j}\right| \leq C \exp \left(M\left(L \lambda_{l}^{1 / \nu}\right)\right) \forall 1 \leq j \leq d_{l}\right\},
$$

where $\Sigma=\left\{v=\left(v_{l}\right)_{l \in \mathbb{N}_{0}}, v_{l} \in \mathbb{C}^{d_{l}}\right\}$ is as in (2.2).

By the diagonal transform we have $D_{L} \cong l^{\infty}$, and since $l^{\infty}$ is a perfect space so we have $\widehat{D_{L}} \cong l^{1}$, and it is given by

$$
\widehat{D_{L}}=\left\{w=\left(w_{l}\right) \in \Sigma: \sum_{l=0}^{\infty} \sum_{j=1}^{d_{l}} \exp \left(M\left(L \lambda_{l}^{1 / \nu}\right)\right)\left|\left(w_{l}\right)_{j}\right|<\infty\right\} .
$$

By Theorem 4.3 we know that $\widehat{\Gamma_{\left\{M_{k}\right\}}(X)}=\bigcap_{L>0} D_{L}$, and hence $\left[\Gamma_{\left\{M_{k}\right\}}(X)\right]^{\wedge}=$ $\bigcup_{L>0} \widehat{D_{L}}$. This means that $w \in\left[\Gamma_{\left\{M_{k}\right\}}(X)\right]^{\wedge}$ if and only if there exists $L_{2}>0$, such that we have

$$
\sum_{l=0}^{\infty} \sum_{j=1}^{d_{l}} \exp \left(M\left(L_{2} \lambda_{l}^{1 / \nu}\right)\right)\left|\left(w_{l}\right)_{j}\right|<\infty .
$$

Let $1 \leq p<q \leq \infty$ and let $a \in \mathbb{C}^{d \times d}$. Then we have the estimates

$$
\|a\|_{\ell^{p}(\mathbb{C})} \leq d^{2\left(\frac{1}{p}-\frac{1}{q}\right)}\|a\|_{\ell^{q}(\mathbb{C})} \quad \text { and } \quad\|a\|_{\ell^{q}(\mathbb{C})} \leq d^{\frac{2}{q}}\|a\|_{\ell^{p}(\mathbb{C})} ;
$$

see, e.g., [3, Lemma 3.2] for a simple proof. In particular, we have $d^{-1}\|a\|_{l^{1}} \leq$ $\|a\|_{\text {HS }} \leq d\|a\|_{l^{1}}$ for $a \in \mathbb{C}^{d \times d}$. Here we also note the estimate: for every $q, L>0$ and $\delta>0$ there exists $C>0$ such that

$$
\lambda_{l}^{q} e^{-\delta M\left(L \lambda_{l}^{1 / \nu}\right)} \leq C
$$

see, e.g., 44, (2.15)]. These estimates and Proposition 2.2 imply

$$
\begin{aligned}
& \sum_{l=0}^{\infty} \exp \left(M\left(L \lambda_{l}^{1 / \nu}\right)\right)\left\|w_{l}\right\|_{\text {HS }} \\
& \quad \leq \sum_{l=0}^{\infty} d_{l} \exp \left(M\left(L \lambda_{l}^{1 / \nu}\right)\right)\left\|w_{l}\right\|_{l^{1}\left(\mathbb{C}^{d_{l}}\right)} \\
& \quad \leq C \sum_{l=0}^{\infty}\left(1+\lambda_{l}\right)^{\frac{n}{\nu}} \exp \left(-M\left(L \lambda_{l}^{1 / \nu}\right)\right) \exp \left(2 M\left(L \lambda_{l}^{1 / \nu}\right)\right)\left\|w_{l}\right\|_{l^{1}\left(\mathbb{C}^{d_{l}}\right)} \\
& \quad \leq C^{\prime} \sum_{l=0}^{\infty} \sum_{j=1}^{d_{l}} \exp \left(2 M\left(L \lambda_{l}^{1 / \nu}\right)\right)\left|\left(w_{l}\right)_{j}\right| \\
& \quad \leq C^{\prime \prime} \sum_{l=0}^{\infty} \sum_{j=1}^{d_{l}} \exp \left(M\left(L_{2} \lambda_{l}^{1 / \nu}\right)\right)\left|\left(w_{l}\right)_{j}\right|<\infty
\end{aligned}
$$


where $L_{2}=L \sqrt{A} H$, where $A, H>0$ in (M.2). The above claim will be true if we can show that $\exp \left(2 M\left(L \lambda_{l}^{1 / \nu}\right)\right) \leq \exp \left(M\left(L_{2} \lambda_{l}^{1 / \nu}\right)\right)$. This follows from property (M.2).

$\Longleftarrow$ : Converse follows similarly using estimates (4.3).

We can now prove Theorem 4.4

Proof of Theorem 4.4. We always have

$$
\Gamma_{\left\{M_{k}\right\}}(X) \subseteq\left[\widehat{\Gamma_{\left\{M_{k}\right\}}(X)}\right]^{\wedge}
$$

from the definition. So we need to prove that $\left[\Gamma_{\left\{M_{k}\right\}}(X)\right]^{\wedge} \subseteq \Gamma_{\left\{M_{k}\right\}}(X)$.

Let $w=\left(w_{l}\right)_{l \in \mathbb{N}_{0}} \in\left[\widehat{\Gamma_{\left\{M_{k}\right\}}(X)}\right]^{\wedge}, w_{l}=\left(w_{l}^{j}\right)_{j=1}^{d_{l}}$, and define

$$
\phi(x):=\sum_{l=0}^{\infty} w_{l} \cdot e_{l}(x)=\sum_{l=0}^{\infty} \sum_{j=1}^{d_{l}} w_{l}^{j} e_{l}^{j}(x),
$$

the series makes sense because of Lemma 4.5 and estimates (4.2) and (4.4). Then we have

$$
\begin{aligned}
\widehat{\phi}(m, k) & =\left(\phi, e_{m}^{k}\right)_{L^{2}} \\
& =\int_{X} \phi(x) \overline{e_{m}^{k}(x)} d x \\
& =\sum_{l=0}^{\infty} \sum_{j=1}^{d_{l}} \int_{X} w_{l}^{j} e_{l}^{j}(x) \overline{e_{m}^{k}(x)} d x \\
& =\sum_{l=0}^{\infty} \sum_{j=1}^{d_{l}} w_{l}^{j} \delta_{l m} \delta_{j k}=w_{m}^{k}, 1 \leq j \leq d_{l}, 1 \leq k \leq d_{m} .
\end{aligned}
$$

This gives

$$
\|\widehat{\phi}(m)\|_{\mathrm{HS}}=\left\|w_{m}\right\|_{\mathrm{HS}} .
$$

Now since $w \in\left[\widehat{\Gamma_{\left\{M_{k}\right\}}(X)}\right]^{\wedge}$, by Lemma 4.5 there exists $L>0$ such that

$$
\sum_{l=0}^{\infty} \exp \left(M\left(L \lambda_{l}^{1 / \nu}\right)\right)\left\|w_{l}\right\|_{\mathrm{HS}}<\infty .
$$

Since $\widehat{\phi}(l)=w_{l}$, it follows that there exists $C>0$ such that

$$
\|\widehat{\phi}(l)\|_{\mathrm{HS}} \leq C \exp \left(-M\left(L \lambda_{l}^{1 / \nu}\right)\right) .
$$

By Theorem 4.1, we have $\phi \in \Gamma_{\left\{M_{k}\right\}}(X)$. Hence we have shown that

$$
\left[\widehat{\Gamma_{\left\{M_{k}\right\}}(X)}\right]^{\wedge} \subseteq \Gamma_{\left\{M_{k}\right\}}(X) .
$$

So we have $\left[\widehat{\Gamma_{\left\{M_{k}\right\}}(X)}\right]^{\wedge}=\Gamma_{\left\{M_{k}\right\}}(X)$, and hence $\Gamma_{\left\{M_{k}\right\}}(X)$ is a perfect space.

Next we proceed to prove the equivalence of two expressions for the duality. 
Lemma 4.6. Let $v \in \Gamma_{\left\{M_{k}\right\}}(X)$ and $w \in \widehat{\Gamma_{\left\{M_{k}\right\}}}(X)$; then

$$
\sum_{k=0}^{\infty}\left\|\left(\widehat{v}_{k}\right)\right\|_{\text {HS }}\left\|\left(w_{k}\right)\right\|_{\text {HS }}<\infty
$$

if and only if

$$
\sum_{k=0}^{\infty} \sum_{i=1}^{d_{k}}\left|\left(\widehat{v_{k}}\right)_{i}\right|\left|\left(w_{k}\right)_{i}\right|<\infty
$$

Proof. $\Longrightarrow$ : The proof is straightforward, following from the estimate

$$
\left(\sum_{i=1}^{d} a_{i} b_{i}\right)^{2} \lesssim\left(\sum_{i=1}^{d} a_{i}^{2}\right)\left(\sum_{i=1}^{d} b_{i}^{2}\right)
$$

$\Longleftarrow$ : We will be using the equality

$$
\left(\sum_{i=1}^{n}\left|a_{i}\right|\right)\left(\sum_{i=1}^{n}\left|b_{i}\right|\right)=\sum_{i=1}^{n}\left|a_{i}\right|\left|b_{i}\right|+\sum_{i=1}^{n}\left|a_{i}\right|\left(\sum_{j=1}^{n}\left|b_{j}\right|-\left|b_{i}\right|\right)
$$

for any $a_{i}, b_{i} \in \mathbb{R}$, yielding

$$
\begin{aligned}
& \left\|\left(\widehat{v}_{k}\right)||_{\mathrm{HS}}\right\|\left(w_{k}\right) \|_{\mathrm{HS}} \\
\leq & \sum_{i=1}^{d_{k}}\left|\left(\widehat{v}_{k}\right)_{i}\right| \sum_{i=1}^{d_{k}}\left|\left(w_{k}\right)_{i}\right| \\
= & \sum_{i=1}^{d_{k}}\left|\left(\widehat{v}_{k}\right)_{i}\right|\left|\left(w_{k}\right)_{i}\right|+\sum_{i=1}^{d_{k}}\left|\left(w_{k}\right)_{i}\right|\left(\sum_{j=1}^{d_{k}}\left|\left(\widehat{v}_{k}\right)_{j}\right|-\left|\left(\widehat{v}_{k}\right)_{i}\right|\right) .
\end{aligned}
$$

We consider the second term in the above inequality, that is,

$$
\left(\sum_{i=1}^{d_{k}}\left|\left(w_{k}\right)_{i}\right|\left(\sum_{j=1}^{d_{k}}\left|\left(\widehat{v}_{k}\right)_{j}\right|-\left|\left(\widehat{v}_{k}\right)_{i}\right|\right)\right) \leq C\left(\sum_{i=1}^{d_{k}}\left|\left(w_{k}\right)_{i}\right|\left(d_{k} e^{-M\left(L \lambda_{k}^{1 / \nu}\right)}\right)\right),
$$

for some $C>0$ and $L>0$. Then using (4.8) in (4.7) we get

$$
\sum_{k=0}^{\infty}\left\|\left(\widehat{v}_{k}\right)\right\|_{\mathrm{HS}}||\left(w_{k}\right) \|_{\mathrm{HS}} \leq \sum_{k=0}^{\infty} \sum_{i=1}^{d_{k}}\left|\left(w_{k}\right)_{i}\right|\left(\left|\left(\widehat{v}_{k}\right)_{i}\right|+C d_{k} e^{-M\left(L \lambda_{k}^{1 / \nu}\right)}\right) .
$$

Now let $\left|\left(\widehat{u}_{k}\right)_{i}\right|=\left|\left(\widehat{v}_{k}\right)_{i}\right|+C d_{k} e^{-M\left(L \lambda_{k}^{1 / \nu}\right)}$, for $i=1,2, \ldots, d_{k}$, and $k \in \mathbb{N}_{0}$. So then we have

$$
\sum_{k=0}^{\infty}\left\|\left(\widehat{v}_{k}\right)\right\|_{\mathrm{HS}}\left\|\left(w_{k}\right)\right\|_{\mathrm{HS}} \leq \sum_{k=0}^{\infty} \sum_{i=1}^{d_{k}}\left|\left(w_{k}\right)_{i}\right|\left|\left(\widehat{u}_{k}\right)_{i}\right| .
$$

Now for some $C^{\prime \prime}>0$ and $L_{2}>0$, we have

$$
\begin{aligned}
\left\|\widehat{u}_{k}\right\|_{\text {HS }} & \left.=\left(\sum_{i=1}^{d_{k}}\left(\left|\left(\widehat{v}_{k}\right)_{i}\right|^{2}+C^{2} d_{k}^{2} e^{-2 M\left(L \lambda_{k}^{1 / \nu}\right)}+2 C d_{k}\left|\left(\widehat{v}_{k}\right)_{i}\right| e^{-M\left(L \lambda_{k}^{1 / \nu}\right)}\right)\right)\right)^{1 / 2} \\
(4.10) \leq C^{\prime \prime} e^{-M\left(L_{2} \lambda_{k}^{1 / \nu}\right)} & \leq
\end{aligned}
$$


i.e., $u \in \Gamma_{\left\{M_{k}\right\}}(X)$. This is true since

$$
\begin{aligned}
& \left(\sum_{i=1}^{d_{k}}\left(\left|\left(\widehat{v}_{k}\right)_{i}\right|^{2}+C^{2} d_{k}^{2} e^{-2 M\left(L \lambda_{k}^{1 / \nu}\right)}+2 C d_{k}\left|\left(\widehat{v}_{k}\right)_{i}\right| e^{-M\left(L \lambda_{k}^{1 / \nu}\right)}\right)\right)^{1 / 2} \\
\leq & \left(\sum_{i=1}^{d_{k}}\left(C^{2} e^{-2 M\left(L \lambda_{k}^{1 / \nu}\right)}+C^{2} d_{k}^{2} e^{-2 M\left(L \lambda_{k}^{1 / \nu}\right)}+2 C^{2} d_{k} e^{-2 M\left(L \lambda_{k}^{1 / \nu}\right)}\right)\right)^{1 / 2} \\
\leq & \left(\sum_{i=1}^{d_{k}} C^{2}\left(1+d_{k}\right)^{2} e^{-2 M\left(L \lambda_{k}^{1 / \nu}\right)}\right)^{1 / 2} \\
\leq & C\left(1+d_{k}\right)^{3 / 2} e^{-M\left(L \lambda_{k}^{1 / \nu}\right)} \\
\leq & C^{\prime} e^{-\frac{1}{2} M\left(L \lambda_{k}^{1 / \nu}\right)} \\
\leq & C^{\prime \prime} e^{-M\left(L_{2} \lambda^{1 / 2}\right)}
\end{aligned}
$$

where $L_{2}=\frac{L}{\sqrt{A} H}$, with $A, H$ are constants in condition (M.2).

Now since $w \in \widehat{\Gamma_{\left\{M_{k}\right\}}}(X)$, so from (4.9) we have

$$
\sum_{k=0}^{\infty}\left\|\left(\widehat{v}_{k}\right)\right\|_{\mathrm{HS}}||\left(w_{k}\right)\left\|_{\mathrm{HS}} \lesssim \sum_{k=0}^{\infty} \sum_{i=1}^{d_{k}}\left|\left(w_{k}\right)_{i} \|\left(\widehat{u}_{k}\right)_{i}\right|<\infty,\right.
$$

completing the proof.

Theorem 4.7 (Adjointness theorem). Let $\left\{M_{k}\right\}$ and $\left\{N_{k}\right\}$ satisfy conditions (M.0)-(M.3). A linear mapping $f: \Gamma_{\left\{M_{k}\right\}}(X) \rightarrow \Gamma_{\left\{N_{k}\right\}}(X)$ is sequential if and only if $f$ is represented by an infinite tensor $\left(f_{k j l i}\right), k, j \in \mathbb{N}_{0}, 1 \leq l \leq d_{j}$ and $1 \leq i \leq d_{k}$ such that for any $u \in \Gamma_{\left\{M_{k}\right\}}(X)$ and $v \in \widehat{\Gamma_{\left\{N_{k}\right\}}}(X)$ we have

$$
\sum_{j=0}^{\infty} \sum_{l=1}^{d_{j}}\left|f_{k j l i}\right||\widehat{u}(j, l)|<\infty \text { for all } k \in \mathbb{N}_{0}, i=1,2, \ldots, d_{k},
$$

and

$$
\sum_{k=0}^{\infty} \sum_{i=1}^{d_{k}}\left|\left(v_{k}\right)_{i}\right|\left|\left(\sum_{j=0}^{\infty} f_{k j} \widehat{u}(j)\right)_{i}\right|<\infty .
$$

Furthermore, the adjoint mapping $\widehat{f}: \widehat{\Gamma_{\left\{N_{k}\right\}}}(X) \rightarrow \widehat{\Gamma_{\left\{M_{k}\right\}}}(X)$ defined by the formula $\widehat{f}(v)=v \circ f$ is also sequential, and the transposed matrix $\left(f_{k j}\right)^{t}$ represents $\widehat{f}$, with $f$ and $\widehat{f}$ related by $\langle f(u), v\rangle=\langle u, \widehat{f}(v)\rangle$.

Let us summarise the ranges for indices in the used notation as well as give more explanation to (4.12). For $f: \Gamma_{\left\{M_{k}\right\}}(X) \rightarrow \Gamma_{\left\{N_{k}\right\}}(X)$ and $u \in \Gamma_{\left\{M_{k}\right\}}(X)$ we write

$$
\mathbb{C}^{d_{k}} \ni f(u)_{k}=\sum_{j=0}^{\infty} f_{k j} \widehat{u}(j)=\sum_{j=0}^{\infty} \sum_{l=1}^{d_{j}} f_{k j l} \widehat{u}(j, l), \quad k \in \mathbb{N}_{0},
$$

so that

$$
f_{k j l} \in \mathbb{C}^{d_{k}}, f_{k j l i} \in \mathbb{C}, \quad k, j \in \mathbb{N}_{0}, 1 \leq l \leq d_{j}, 1 \leq i \leq d_{k},
$$


and

$$
\mathbb{C} \ni\left(f(u)_{k}\right)_{i}=f(u)_{k i}=\sum_{j=0}^{\infty} \sum_{l=1}^{d_{j}} f_{k j l i} \widehat{u}(j, l), \quad k \in \mathbb{N}_{0}, 1 \leq i \leq d_{k},
$$

where we view $f_{k j}$ as a matrix, $f_{k j} \in \mathbb{C}^{d_{k} \times d_{j}}$, and the product of the matrices has been explained in (4.13).

Remark 4.8. Let us now briefly describe how the tensor $\left(f_{k j l i}\right), k, j \in \mathbb{N}_{0}, 1 \leq l \leq d_{j}$, $1 \leq i \leq d_{k}$, is constructed given a sequential mapping $f: \Gamma_{\left\{M_{k}\right\}}(X) \rightarrow \Gamma_{\left\{N_{k}\right\}}(X)$. For every $k \in \mathbb{N}_{0}$ and $1 \leq i \leq d_{k}$, define the family $v^{k i}=\left(v_{j}^{k i}\right)_{j \in \mathbb{N}_{0}}$ such that each $v_{j}^{k i} \in \mathbb{C}^{d_{j}}$ is defined by

$$
v_{j}^{k i}(l)=\left\{\begin{array}{l}
1, j=k, l=i \\
0 \text { otherwise }
\end{array}\right.
$$

Then $v^{k i} \in\left[\Gamma_{\left\{N_{k}\right\}}(X)\right]^{\wedge}$, and since $f$ is sequential we have $v^{k i} \circ f \in\left[\Gamma_{\left\{M_{k}\right\}}(X)\right]^{\wedge}$, and we can write $v^{k i} \circ f=\left(v^{k i} \circ f\right)_{j \in \mathbb{N}_{0}}$, where $\left(v^{k i} \circ f\right)_{j} \in \mathbb{C}^{d_{j}}$. Then for each $1 \leq l \leq d_{j}$ we set

$$
f_{k j l i}:=\left(v^{k i} \circ f\right)_{j}(l),
$$

the $l^{t h}$ component of the vector $\left(v^{k i} \circ f\right)_{j} \in \mathbb{C}^{d_{j}}$. The formula (4.17) will be shown in the proof of Theorem 4.7. In particular, since for $\phi \in \Gamma_{\left\{M_{k}\right\}}(X)$ we have $f(\phi) \in$ $\Gamma_{\left\{N_{k}\right\}}(X)$, it will be a consequence of (4.28) and (4.29) later on that

$$
v^{k i} \circ f(\phi)=(\widehat{f(\phi)})(k, i)=\sum_{j=0}^{\infty} \sum_{l=1}^{d_{j}} f_{k j l i} \widehat{\phi}(j, l),
$$

so that the tensor $\left(f_{k j l i}\right)$ is describing the transformation of the Fourier coefficients of $\phi$ into those of $f(\phi)$.

Another meaning of condition (4.11) is that if for each $k \in \mathbb{N}_{0}$ and $1 \leq i \leq d_{k}$ we define

$$
f^{k i}(j, l):=f_{k j l i},
$$

then $f^{k i} \in\left[\Gamma_{\left\{M_{k}\right\}}(X)\right]^{\wedge}$. Condition (4.12) is the continuity condition saying that for every $u \in \Gamma_{\left\{M_{k}\right\}}(X)$ we have that

$$
\sum_{j=0}^{\infty} \sum_{l=1}^{d_{j}} f_{k j l i} \widehat{u}(j, l) \in \Gamma_{\left\{N_{k}\right\}}(X) .
$$

To prove Theorem 4.7 we first establish the following lemma.

Lemma 4.9. Let $\left(f_{k j l i}\right)_{k, j \in \mathbb{N}_{0}, 1 \leq l \leq d_{j}, 1 \leq i \leq d_{k}}$ be an infinite tensor satisfying (4.11) and (4.12). Then for any $u \in \Gamma_{\left\{M_{k}\right\}}(X)$ and $v \in\left[\Gamma_{\left\{N_{k}\right\}}(X)\right]^{\wedge}$, we have

$$
\lim _{n \rightarrow \infty} \sum_{k=0}^{\infty} \sum_{i=1}^{d_{k}}\left|\left(v_{k}\right)_{i}\right|\left|\left(\sum_{0 \leq j \leq n} f_{k j} \widehat{u}(j)\right)_{i}\right|=\sum_{k=0}^{\infty} \sum_{i=1}^{d_{k}}\left|\left(v_{k}\right)_{i}\right|\left|\left(\sum_{j=0}^{\infty} f_{k j} \widehat{u}(j)\right){ }_{i}\right| .
$$

Proof of Lemma 4.9, Let $u \in \Gamma_{\left\{M_{k}\right\}}(X)$ and $u \approx(\widehat{u}(l))_{l \in \mathbb{N}_{0}}$. Define

$$
u^{n}:=\left(\widehat{u}^{(n)}(l)\right)_{l \in \mathbb{N}_{0}}
$$


by setting

$$
\widehat{u}^{(n)}(l)= \begin{cases}\widehat{u}(l), & l \leq n, \\ 0, & l>n .\end{cases}
$$

Then for any $w \in \widehat{\Gamma_{\left\{M_{k}\right\}}}(X),\left\langle u-u^{n}, w\right\rangle \rightarrow 0$ as $n \rightarrow \infty$. This is true since $\sum_{l=0}^{\infty}\left|\widehat{u}(l) \cdot w_{l}\right|<\infty$ so that

$$
\left|\left\langle u-u^{n}, w\right\rangle\right| \leq \sum_{l \geq n}\left|\widehat{u_{l}} \cdot w_{l}\right| \rightarrow 0
$$

as $n \rightarrow \infty$. Now for any $u \in \Gamma_{\left\{M_{k}\right\}}(X)$ and $v \in\left[\Gamma_{\left\{N_{k}\right\}}(X)\right]^{\wedge}$ and from (4.11) and (4.12) we have

$$
\begin{gathered}
\langle f(u), v\rangle=\sum_{k=0}^{\infty}(f(u))_{k} \cdot v_{k}=\sum_{k=0}^{\infty}\left(\sum_{j=0}^{\infty} f_{k j} \widehat{u}(j)\right) \cdot v_{k} \\
=\sum_{k=0}^{\infty} \sum_{j=0}^{\infty} \sum_{\ell=1}^{d_{j}} \sum_{i=1}^{d_{k}} f_{k j \ell i} \widehat{u}(j, \ell)\left(v_{k}\right)_{i}=\sum_{j=0}^{\infty} \sum_{\ell=1}^{d_{j}} \widehat{u}(j, \ell) \sum_{k=0}^{\infty} \sum_{i=1}^{d_{k}} f_{k j \ell i}\left(v_{k}\right)_{i} \\
=\sum_{j=0}^{\infty} \sum_{\ell=1}^{d_{j}} \widehat{u}(j, \ell) \sum_{k=0}^{\infty} f_{k j \ell} \cdot v_{k}=\sum_{j=0}^{\infty} \widehat{u}(j) \cdot(v \circ f)_{j}=\langle u, v \circ f\rangle,
\end{gathered}
$$

where

$$
\mathbb{C}^{d_{j}} \ni(v \circ f)_{j}=\left\{\sum_{k=0}^{\infty} f_{k j \ell} \cdot v_{k}\right\}_{\ell=1}^{d_{j}}, \quad j \in \mathbb{N}_{0},
$$

and

$$
v \circ f=\left\{(v \circ f)_{j}\right\}_{j=0}^{\infty} .
$$

For sequential mapping $f, v \circ f \in\left[\Gamma_{\left\{M_{k}\right\}}(X)\right]^{\wedge}$ and

$$
\sum_{j=0}^{\infty} u(j) \cdot(v \circ f)_{j}=\langle u, v \circ f\rangle=(v \circ f)(u),
$$

so that we can write $(v \circ f) \in \mathbb{C}^{d_{j}}$ and also $(v \circ f)(u)=\langle v, f(u)\rangle$. So for any $u \approx(\widehat{u}(j))_{j \in \mathbb{N}_{0}} \in \Gamma_{\left\{M_{k}\right\}}(X)$ from the definition of $\left[\Gamma_{\left\{M_{k}\right\}}(X)\right]^{\wedge}$ we have

$$
\sum_{j \in \mathbb{N}_{0}} \sum_{l=1}^{d_{j}}\left|(v \circ f)_{j l}\right||\widehat{u}(j, l)|<\infty .
$$

Hence the series $\sum_{j=0}^{\infty}\left|(v \circ f)_{j} \cdot \widehat{u}(j)\right|$ is convergent.

We can then conclude that $v \circ f \in\left[\Gamma_{\left\{M_{k}\right\}}(X)\right]^{\wedge}$ and we have

$$
\left\langle f(u)-f\left(u^{n}\right), v\right\rangle=\left\langle u-u^{n}, v \circ f\right\rangle \rightarrow 0
$$

as $n \rightarrow \infty$. Therefore,

$$
\langle f(u), v\rangle=\lim _{n \rightarrow \infty}\left\langle f\left(u^{n}\right), v\right\rangle
$$


for all $u \in \Gamma_{\left\{M_{k}\right\}}(X)$ and $v \in\left[\Gamma_{\left\{N_{k}\right\}}(X)\right]^{\wedge}$. Hence for any $u \in \Gamma_{\left\{M_{k}\right\}}(X)$ and $v \in\left[\Gamma_{\left\{N_{k}\right\}}(X)\right]^{\wedge}$ we have

$$
\lim _{n \rightarrow \infty} \sum_{k=0}^{\infty} v_{k} \cdot\left(\sum_{0 \leq j \leq n} f_{k j} \widehat{u}(j)\right)=\sum_{k=0}^{\infty} v_{k} \cdot\left(\sum_{j=0}^{\infty} f_{k j} \widehat{u}(j)\right),
$$

that is,

$$
\lim _{n \rightarrow \infty} \sum_{k=0}^{\infty} \sum_{i=1}^{d_{k}}\left(v_{k}\right)_{i}\left(\sum_{j \leq n} f_{k j} \widehat{u}(j)\right)_{i}=\sum_{k=0}^{\infty} \sum_{i=1}^{d_{k}}\left(v_{k}\right)_{i}\left(\sum_{j=0}^{\infty} f_{k j} \widehat{u}(j)\right)_{i} .
$$

Now we will use the fact that if $u \in \Gamma_{\left\{M_{k}\right\}}(X)$, then $|u| \in \Gamma_{\left\{M_{k}\right\}}(X)$ where $|u|=$ $\left(\left.\widehat{\mid u}\right|_{j}\right)_{j \in \mathbb{N}_{0}},\left.\widehat{|u|}\right|_{j} \in \mathbb{R}^{d_{j}}$, with

$$
\widehat{|u|}_{j}:=\left[\begin{array}{c}
|\widehat{u}(j, 1)| \\
|\widehat{u}(j, 2)| \\
\vdots \\
\left|\widehat{u}\left(j, d_{j}\right)\right|
\end{array}\right]
$$

in view of the Theorem 4.4. The same is true for the dual space $\left[\Gamma_{\left\{N_{k}\right\}}(X)\right]^{\wedge}$. So then this argument gives

$$
\lim _{n \rightarrow \infty} \sum_{k=0}^{\infty} \sum_{i=1}^{d_{k}}\left|\left(v_{k}\right)_{i}\right|\left|\left(\sum_{0 \leq j \leq n} f_{k j} \widehat{u}(j)\right)\right|=\sum_{k=0}^{\infty} \sum_{i=1}^{d_{k}}\left|\left(v_{k}\right)_{i}\right|\left|\left(\sum_{j=0}^{\infty} f_{k j} \widehat{u}(j)\right){ }_{i}\right| .
$$

The proof is complete.

Proof of Theorem 4.7, Let us assume first that the mapping $f: \Gamma_{\left\{M_{k}\right\}}(X) \rightarrow$ $\Gamma_{\left\{N_{k}\right\}}(X)$ can be represented by $f=\left(f_{k j l i}\right)_{k, j \in \mathbb{N}_{0}, 1 \leq l \leq d_{j}, 1 \leq i \leq d_{k}}$, an infinite tensor such that

$$
\sum_{j=0}^{\infty} \sum_{l=1}^{d_{j}}\left|f_{k j l i}\right||\widehat{u}(j, l)|<\infty \text { for all } k \in \mathbb{N}_{0}, i=1,2, \ldots, d_{k},
$$

and

$$
\sum_{k=0}^{\infty} \sum_{i=1}^{d_{k}}\left|\left(v_{k}\right)_{i}\right|\left|\left(\sum_{j=0}^{\infty} f_{k j} \widehat{u}(j)\right)_{i}\right|<\infty
$$

hold for all $u \in \Gamma_{\left\{M_{k}\right\}}(X)$ and $v \in\left[\Gamma_{\left\{N_{k}\right\}}(X)\right]^{\wedge}$.

Let $\widehat{u}_{1}=\left(\widehat{u_{1}}(p)\right)_{p \in \mathbb{N}_{0}}$ be such that for some $j, l$ where $j \in \mathbb{N}_{0}, 1 \leq l \leq d_{j}$ we have

$$
\widehat{u_{1}}(p, q)=\left\{\begin{array}{l}
1, p=j, q=l \\
0 \text { otherwise }
\end{array}\right.
$$


Then $u_{1} \in \Gamma_{\left\{M_{k}\right\}}(X)$ so $f u_{1}=f\left(u_{1}\right) \in \Gamma_{\left\{N_{k}\right\}}(X)$ and

$$
\begin{aligned}
\left(f u_{1}\right)_{k} & =\sum_{p=0}^{\infty} f_{k p} \widehat{u}_{1}(p) \\
& =\sum_{p=0}^{\infty} \sum_{q=1}^{d_{p}} f_{k p q} \widehat{u_{1}}(p, q) \\
& =\sum_{q=1}^{d_{j}} f_{k j q} \widehat{u_{1}}(j, q) \\
& =f_{k j l} \in \mathbb{C}^{d_{k}} .
\end{aligned}
$$

We now first show that

$$
\widehat{(f u)}(k)=\sum_{j=0}^{\infty} \sum_{l=1}^{d_{j}} f_{k j l} \widehat{u}(j, l),
$$

where $f_{k j l i} \in \mathbb{C}$ for each $k, j \in \mathbb{C}, 1 \leq l \leq d_{j}$ and $1 \leq i \leq d_{k}$. The way in which $f$ has been defined we have

$$
(f u)_{k}=\sum_{j=0}^{\infty} \sum_{l=1}^{d_{j}} f_{k j l} \widehat{u}(j, l), \quad f_{k j l} \in \mathbb{C}^{d_{k}} .
$$

Also since $u \in \Gamma_{\left\{M_{k}\right\}}(X)$, from our assumption we have $f u \in \Gamma_{\left\{N_{k}\right\}}(X)$ and $f u \approx$ $(\widehat{(f u)}(j))_{j \in \mathbb{N}_{0}}$ so $(f u)_{k} \approx \widehat{(f u)}(k)$.

We can then write $\widehat{(f u)}(k)=\sum_{j} \sum_{l=1}^{d_{j}} f_{k j l} \widehat{u}(j, l)$. Since we know that $v \in$ $\left[\Gamma_{\left\{N_{k}\right\}}(X)\right]^{\wedge}$ and $f u \in \Gamma_{\left\{N_{k}\right\}}(X)$, we have

$$
\sum_{k=0}^{\infty} \sum_{i=1}^{d_{k}}\left|\left(v_{k}\right)_{i}\right|\left|(\widehat{(f u)}(k))_{i}\right|=\sum_{k=0}^{\infty} \sum_{i=1}^{d_{k}}\left|\left(v_{k}\right)_{i} \| \sum_{j=0}^{\infty} \sum_{l=1}^{d_{j}} f_{k j l i} \widehat{u}(j, l)\right|<\infty .
$$

In particular using the definition of $u_{1}$ and (4.22) we get

$$
\sum_{k=0}^{\infty} \sum_{i=1}^{d_{k}}\left|\left(v_{k}\right)_{i}\right|\left|\sum_{p=0}^{\infty} \sum_{q=1}^{d_{k}} f_{k p q i} \widehat{u_{1}}(p, q)\right|=\sum_{k=0}^{\infty} \sum_{i=1}^{d_{k}}\left|\left(v_{k}\right)_{i}\right|\left|f_{k j l i}\right|<\infty
$$

for any $j \in \mathbb{N}_{0}$ and $1 \leq l \leq d_{j}$.

Now for any $u \in \Gamma_{\left\{M_{k}\right\}}(X)$ consider

$$
J=\sum_{j=0}^{\infty} \sum_{l=1}^{d_{j}}\left|\sum_{k=0}^{\infty} \sum_{i=1}^{d_{k}}\left(v_{k}\right)_{i} f_{k j l i} \| \widehat{u}(j, l)\right| .
$$

Then we consider the series

$$
I_{n}:=\sum_{j \leq n} \sum_{l=1}^{d_{j}}\left|\sum_{k=0}^{\infty} \sum_{i=1}^{d_{k}}\left(v_{k}\right)_{i} f_{k j l i}\right||\widehat{u}(j, l)|,
$$


so that we have

$$
\begin{aligned}
I_{n} & =\sum_{j \leq n} \sum_{l=1}^{d_{j}}\left|\sum_{k=0}^{\infty} \sum_{i=1}^{d_{k}}\left(v_{k}\right)_{i} f_{k j l i}\right||\widehat{u}(j, l)| \\
& =\sum_{j \leq n} \sum_{l=1}^{d_{j}}\left|\sum_{k=0}^{\infty} \sum_{i=1}^{d_{k}}\left(v_{k}\right)_{i} f_{k j l i} \widehat{u}(j, l)\right| .
\end{aligned}
$$

Let $\epsilon=\left(\epsilon_{i}\right)_{1 \leq i \leq d_{k}}, k \in \mathbb{N}_{0}$, be such that $\epsilon_{i} \in \mathbb{C}$ and $\left|\epsilon_{i}\right|=1$ for all $i$ and such that

$$
\left.\mid \sum_{k=0}^{\infty} \sum_{i=1}^{d_{k}}\left(v_{k}\right)_{i} f_{k j l i}\right) \widehat{u}(j, l) \mid=\sum_{k=0}^{\infty} \sum_{i=1}^{d_{k}}\left(v_{k}\right)_{i} f_{k j l i} \widehat{u}(j, l) \epsilon_{i} .
$$

Then

$$
\begin{aligned}
I_{n} & =\sum_{j \leq n} \sum_{l=1}^{d_{j}} \sum_{k=0}^{\infty} \sum_{i=1}^{d_{k}}\left(v_{k}\right)_{i} f_{k j l i} \widehat{u}(j, l) \epsilon_{i} \\
& \left.\leq \sum_{k=0}^{\infty} \sum_{i=1}^{d_{k}}\left|\left(v_{k}\right)_{i}\right| \mid \sum_{j \leq n} \sum_{l=1}^{d_{j}} f_{k j l i}\right) \widehat{u}(j, l) \epsilon_{i} \mid .
\end{aligned}
$$

It follows from Lemma 4.9 that

$$
\begin{aligned}
\lim _{n \rightarrow \infty} \sum_{k=0}^{\infty} \sum_{i=1}^{d_{k}}\left|\left(v_{k}\right)_{i}\right|\left|\sum_{j \leq n} \sum_{l=1}^{d_{j}} f_{k j l i} \widehat{u}(j, l) \epsilon_{i}\right| \\
=\sum_{k=0}^{\infty} \sum_{i=1}^{d_{k}}\left|\left(v_{k}\right)_{i}\right|\left|\sum_{j=0}^{\infty} \sum_{l=1}^{d_{j}} f_{k j l i} \widehat{u}(j, l) \epsilon_{i}\right|<\infty .
\end{aligned}
$$

Then

$$
J=\sum_{j=0}^{\infty} \sum_{l=1}^{d_{j}}\left|\sum_{k=0}^{\infty} \sum_{i=1}^{d_{k}}\left(v_{k}\right)_{i} f_{k j l i}\right||\widehat{u}(j, l)|<\infty .
$$

So we proved that if $\left(f_{k j l i}\right)$ satisfies

- $\sum_{j=0}^{\infty} \sum_{l=1}^{d_{j}}\left|f_{k j l i}\right||\widehat{u}(j, l)|<\infty$,

- $\sum_{k=0}^{\infty} \sum_{i=1}^{d_{k}}\left|\left(v_{k}\right)_{i}\right|\left|\left(\sum_{j=0}^{\infty} f_{k j} \widehat{u}(j)\right)_{i}\right|<\infty$,

then for any $u \in \Gamma_{\left\{M_{k}\right\}}(X)$ and $v \in\left[\Gamma_{\left\{N_{k}\right\}}(X)\right]^{\wedge}$ we have from (4.23) and (4.25), respectively, that is,

(i) $\sum_{k=0}^{\infty} \sum_{i=1}^{d_{k}}\left|\left(v_{k}\right)_{i}\right|\left|f_{k j l i}\right|<\infty$,

(ii) $\left.\sum_{j=0}^{\infty} \sum_{l=1}^{d_{j}} \mid \sum_{k=0}^{\infty} \sum_{i=1}^{d_{k}}\left(v_{k}\right)_{i} f_{k j l i}\right)|| \widehat{u}(j, l) \mid<\infty$.

Now recall that for $f: \Gamma_{\left\{M_{k}\right\}}(X) \rightarrow \Gamma_{\left\{N_{k}\right\}}(X)$ we have

$$
(f(u))_{k}=\sum_{j=0}^{\infty} \sum_{l=1}^{d_{j}} f_{k j l} \widehat{u}(j, l)
$$


for any $u \in \Gamma_{\left\{M_{k}\right\}}(X)$; then for any $v \in\left[\Gamma_{\left\{N_{k}\right\}}(X)\right]^{\wedge}$, the composed mapping $v \circ f: \Gamma_{\left\{M_{k}\right\}}(X) \rightarrow \mathbb{C}$ is given by

$$
\begin{aligned}
(v \circ f)(u) & =\sum_{k=0}^{\infty} v_{k} \cdot(f(u))_{k}=\sum_{k=0}^{\infty} \sum_{i=1}^{d_{k}}\left(v_{k}\right)_{i}\left(\sum_{j=0}^{\infty} \sum_{l=1}^{d_{j}} f_{k j l i} \widehat{u}(j, l)\right) \\
& =\sum_{j=0}^{\infty} \sum_{l=1}^{d_{j}}\left(\sum_{k=0}^{\infty} \sum_{i=1}^{d_{k}}\left(v_{k}\right)_{i} f_{k j l i}\right) \widehat{u}(j, l) .
\end{aligned}
$$

So by (ii) we get that

$$
|(v \circ f)(u)| \leq \sum_{j=0}^{\infty} \sum_{l=1}^{d_{j}}\left|\sum_{k=0}^{\infty} \sum_{i=1}^{d_{k}}\left(v_{k}\right)_{i} f_{k j l i}\right||\widehat{u}(j, l)|<\infty .
$$

So $\widehat{f}(v)=\left(\widehat{f}(v)_{j l}\right)_{j \in \mathbb{N}, 1 \leq l \leq d_{j}}$, with $\widehat{f}(v)_{j l}=\sum_{k=0}^{\infty} \sum_{i=1}^{d_{k}}\left(v_{k}\right)_{i} f_{k j l i} \in\left[\Gamma_{\left\{M_{k}\right\}}(X)\right]^{\wedge}$ (from the definition of $\left[\Gamma_{\left\{M_{k}\right\}}(X)\right]^{\wedge}$ ), that is, $f$ is sequential. Then $\langle f(u), v\rangle=$ $\langle u, \widehat{f}(v)\rangle$ is also true.

Now to prove the converse part we assume that $f: \Gamma_{\left\{M_{k}\right\}}(X) \rightarrow \Gamma_{\left\{N_{k}\right\}}(X)$ is sequential. We have to show that $f$ can be represented as $f \approx\left(f_{k j l i}\right)_{k, j \in \mathbb{N}_{0}, 1 \leq l \leq d_{j}, 1 \leq i \leq d_{k}}$ and satisfies (4.11) and (4.12).

Define for $k, i$ where $k \in \mathbb{N}_{0}$ and $1 \leq i \leq d_{k}$, the sequence $u^{k i}=\left(u_{j}^{k i}\right)_{j \in \mathbb{N}_{0}}$ such that $u_{j}^{k i} \in \mathbb{C}^{d_{j}}$ and $u_{j}^{k i}(l)=\widehat{u^{k i}}(j, l)$, given by

$$
u_{j}^{k i}(l)=\widehat{u^{k i}}(j, l)=\left\{\begin{array}{l}
1, j=k, l=i, \\
0 \text { otherwise }
\end{array}\right.
$$

Then $u^{k i} \in\left[\Gamma_{\left\{N_{k}\right\}}(X)\right]^{\wedge}$. Now since $f$ is sequential we have $u^{k i} \circ f \in\left[\Gamma_{\left\{M_{k}\right\}}(X)\right]^{\wedge}$ and $u^{k i} \circ f=\left(u^{k i} \circ f\right)_{j \in \mathbb{N}_{0}}$, where $\left(u^{k i} \circ f\right)_{j} \in \mathbb{C}^{d_{j}}$. We denote $u^{k i} \circ f=\left(f_{j}^{k i}\right)_{j \in \mathbb{N}_{0}}$, where $f_{j}^{k i}=\left(u^{k i} \circ f\right)_{j}$. Then $\left(f_{j}^{k i}\right)_{j \in \mathbb{N}_{0}} \in\left[\Gamma_{\left\{M_{k}\right\}}(X)\right]^{\wedge}$ and $f_{j}^{k i} \in \mathbb{C}^{d_{j}}$.

Then for any $\phi \approx(\widehat{\phi}(j))_{j \in \mathbb{N}_{0}} \in \Gamma_{\left\{M_{k}\right\}}(X)$ we have

$$
\sum_{j=0}^{\infty} \sum_{l=1}^{d_{j}}\left|f_{j l}^{k i}\right||\widehat{\phi}(j, i)|<\infty
$$

For $\phi \in \Gamma_{\left\{M_{k}\right\}}(X)$ we can write $f(\phi) \in \Gamma_{\left\{N_{k}\right\}}(X)$. We can write

$$
f(\phi)=\left((f(\phi))^{\wedge}(p)\right)_{p \in \mathbb{N}_{0}} .
$$

So

$$
\begin{aligned}
u^{k i} \circ f(\phi) & \left.=\sum_{j=0}^{\infty} u_{j}^{k i} \widehat{(f(\phi)}\right)_{j} \\
& =\sum_{j=0}^{\infty} \sum_{l=1}^{d_{j}} u_{j l}^{k i} \widehat{(f(\phi))}(j, l) \\
& =(\widehat{f(\phi)})(k, i)\left(\text { from the definition of } u^{k i}\right) .
\end{aligned}
$$


We have $u^{k i} \circ f=\left(f^{k i}\right)_{j} \in\left[\Gamma_{\left\{M_{k}\right\}(X)}\right]^{\wedge}$, so

$$
\begin{aligned}
\left(u^{k i} \circ f\right)(\phi) & =\sum_{j=0}^{\infty} f_{j}^{k i} \widehat{\phi}(j) \\
& =\sum_{j=0}^{\infty} \sum_{l=1}^{d_{j}} f_{j l}^{k i} \widehat{\phi}(j, l) .
\end{aligned}
$$

From (4.28) and (4.29) we have $(\widehat{f(\phi)})(k, i)=\sum_{j=0}^{\infty} \sum_{l=1}^{d_{j}} f_{j l}^{k i} \widehat{\phi}(j, l)$.

Hence $(f(\phi))_{k i}=\sum_{j=0}^{\infty} \sum_{l=1}^{d_{j}} f_{j l}^{k i} \widehat{\phi}(j, l), k \in \mathbb{N}_{0}$, and $1 \leq i \leq d_{k}$, that is, $f$ is represented by the tensor $\left\{\left(f_{j l}^{k i}\right)\right\}_{k, j \in \mathbb{N}_{0}, 1 \leq i \leq d_{k}, 1 \leq l \leq d_{j}}$.

If we denote $f_{j l}^{k i}$ by $f_{j l}^{k i}=f_{k j l i}$, we can say that $f$ is represented by the tensor $\left(f_{k j l i}\right)_{k, j \in \mathbb{N}_{0}, 1 \leq l \leq d_{j}, 1 \leq i \leq d_{k}}$. Also let $v \in\left[\widehat{\Gamma_{\left\{N_{k}\right\}}(X)}\right]$. Since $f(\phi) \in \Gamma_{\left\{N_{k}\right\}}(X)$ for $\phi \in \Gamma_{\left\{M_{k}\right\}}(X)$, then from the definition of $\left[\Gamma_{\left\{N_{k}\right\}}(X)\right]$ we have

$$
\sum_{k=0}^{\infty} \sum_{i=1}^{d_{k}}\left|\left(v_{k}\right)_{i}\right| \sum_{j=0}^{\infty} \sum_{l=1}^{d_{j}} f_{k j l i} \widehat{\phi}(j, l) \mid<\infty .
$$

This completes the proof of Theorem 4.7

\section{Beurling Class of ultradifferentiable FunCtions AND ULTRADISTRIBUTIONS}

In this section we briefly summarise the counterparts of the results of the previous section for the case of Komatsu classes of Beurling type ultradifferentiable functions and ultradistributions. For more details we refer to [4 for a more detailed description of these spaces as well as of their duals and $\alpha$-duals in the sense of Köthe.

The class $\Gamma_{\left(M_{k}\right)}(X)$ is the space of $C^{\infty}$ functions $\phi$ on $X$ such that for every $h>0$ there exists $C_{h}>0$ such that we have

$$
\left\|E^{k} \phi\right\|_{L^{2}(X)} \leq C_{h} h^{\nu k} M_{\nu k}, k=0,1,2, \ldots
$$

For these spaces, we replace condition (M.3) by condition

$\left(\right.$ M. $\left.3^{\prime}\right) \quad$ for every $l>0$ there exists $C_{l}>0$ such that $k ! \leq C_{l} l^{k} M_{k}$ for all $k \in \mathbb{N}_{0}$.

The counterpart of [4, Theorem 4.1 and Theorem 4.3, holds for this class as well, namely, we have

Theorem 5.1. Assume conditions (M.0), (M.1), (M.2), (M.3'). We have $\phi \in$ $\Gamma_{\left(M_{k}\right)}(X)$ if and only if for every $L>0$ there exists $C_{L}>0$ such that

$$
\|\widehat{\phi}(l)\|_{\mathrm{HS}} \leq C_{L} \exp \left\{-M\left(L \lambda_{l}^{1 / \nu}\right)\right\} \quad \text { for all } l \geq 1 .
$$

For the dual space and for the $\alpha$-dual, the following statements are equivalent:

(i) $v \in \Gamma_{\left(M_{k}\right)}^{\prime}(X)$;

(ii) $v \in\left[\Gamma_{\left(M_{k}\right)}(X)\right]^{\wedge}$;

(iii) there exists $L>0$ such that we have

$$
\sum_{l=0}^{\infty} \exp \left(-M\left(L \lambda_{l}^{1 / \nu}\right)\right)\left\|v_{l}\right\|_{\text {HS }}<\infty ;
$$


(iv) there exists $L>0$ and $K>0$ such that

$$
\left\|v_{l}\right\|_{\mathrm{HS}} \leq K \exp \left(M\left(L \lambda_{l}^{1 / \nu}\right)\right)
$$

holds for all $l \in \mathbb{N}_{0}$.

Again we note that given this characterisation of $\alpha$-duals, one can prove that they are perfect, in a way similar to the proof of Theorem 4.4. namely, that

$$
\left[\Gamma_{\left(M_{k}\right)}(X)\right]=\left(\left[\Gamma_{\left(M_{k}\right)}(X)\right]^{\wedge}\right)^{\wedge} .
$$

Finally we can state the counterpart of the adjointness Theorem 4.7

Theorem 5.2 (Adjointness theorem Beurling case). Let $\left\{M_{k}\right\}$ and $\left\{N_{k}\right\}$ satisfy conditions (M.0)-(M.3'). A linear mapping $f: \Gamma_{\left(M_{k}\right)}(X) \rightarrow \Gamma_{\left(N_{k}\right)}(X)$ is sequential if and only if $f$ is represented by an infinite tensor $\left(f_{k j l i}\right), k, j \in \mathbb{N}_{0}, 1 \leq l \leq d_{j}$ and $1 \leq i \leq d_{k}$ such that for any $u \in \Gamma_{\left(M_{k}\right)}(X)$ and $v \in \widehat{\Gamma_{\left(N_{k}\right)}}(X)$ we have

$$
\sum_{j=0}^{\infty} \sum_{l=1}^{d_{j}}\left|f_{k j l i}\right||\widehat{u}(j, l)|<\infty \text { for all } k \in \mathbb{N}_{0}, i=1,2, \ldots, d_{k},
$$

and

$$
\sum_{k=0}^{\infty} \sum_{i=1}^{d_{k}}\left|\left(v_{k}\right)_{i}\right|\left|\left(\sum_{j=0}^{\infty} f_{k j} \widehat{u}(j)\right)_{i}\right|<\infty .
$$

Furthermore, the adjoint mapping $\widehat{f}: \widehat{\Gamma_{\left(N_{k}\right)}}(X) \rightarrow \widehat{\Gamma_{\left(M_{k}\right)}}(X)$ defined by the formula $\widehat{f}(v)=v \circ f$ is also sequential, and the transposed matrix $\left(f_{k j}\right)^{t}$ represents $\widehat{f}$, with $f$ and $\widehat{f}$ related by $\langle f(u), v\rangle=\langle u, \widehat{f}(v)\rangle$.

The proof of Theorem 5.2 is similar to the corresponding proof in Theorem 4.7 for the spaces $\Gamma_{\left\{M_{k}\right\}}(X)$, so we omit the repetition.

\section{REFERENCES}

[1] Richard D. Carmichael, Andrzej Kamiński, and Stevan Pilipović, Boundary values and convolution in ultradistribution spaces, Series on Analysis, Applications and Computation, vol. 1, World Scientific Publishing Co. Pte. Ltd., Hackensack, NJ, 2007. MR.2347838

[2] Antoine Delcroix, Maximilian F. Hasler, Stevan Pilipović, and Vincent Valmorin, Embeddings of ultradistributions and periodic hyperfunctions in Colombeau type algebras through sequence spaces, Math. Proc. Cambridge Philos. Soc. 137 (2004), no. 3, 697-708, DOI 10.1017/S0305004104007923. MR2103924

[3] Aparajita Dasgupta and Michael Ruzhansky, Gevrey functions and ultradistributions on compact Lie groups and homogeneous spaces, Bull. Sci. Math. 138 (2014), no. 6, 756-782, DOI 10.1016/j.bulsci.2013.12.001. MR 3251455

[4] Aparajita Dasgupta and Michael Ruzhansky, Eigenfunction expansions of ultradifferentiable functions and ultradistributions, Trans. Amer. Math. Soc. 368 (2016), no. 12, 8481-8498, DOI 10.1090/tran/6765. MR.3551578

[5] J. Delgado and M. Ruzhansky, Fourier multipliers, symbols and nuclearity on compact manifolds, J. Anal. Math, to appear, arXiv:1404.6479, 2014.

[6] Lars Hörmander, The spectral function of an elliptic operator, Acta Math. 121 (1968), 193218, DOI 10.1007/BF02391913. MR0609014

[7] Julio Delgado and Michael Ruzhansky, Schatten classes on compact manifolds: kernel conditions, J. Funct. Anal. 267 (2014), no. 3, 772-798, DOI 10.1016/j.jfa.2014.04.016. MR3212723 
[8] Claudia Garetto and Michael Ruzhansky, Wave equation for sums of squares on compact Lie groups, J. Differential Equations 258 (2015), no. 12, 4324-4347, DOI 10.1016/j.jde.2015.01.034. MR3327555

[9] Todor Gramchev, Stevan Pilipovic, and Luigi Rodino, Eigenfunction expansions in $\mathbb{R}^{n}$, Proc. Amer. Math. Soc. 139 (2011), no. 12, 4361-4368, DOI 10.1090/S0002-9939-2011-11022-0. MR2823081

[10] Gottfried Köthe, Topological vector spaces. I, Translated from the German by D. J. H. Garling. Die Grundlehren der mathematischen Wissenschaften, Band 159, Springer-Verlag New York Inc., New York, 1969. MR0248498

[11] Hikosaburo Komatsu, Ultradistributions. I. Structure theorems and a characterization, J. Fac. Sci. Univ. Tokyo Sect. IA Math. 20 (1973), 25-105. MR0320743

[12] Hikosaburo Komatsu, Ultradistributions. II. The kernel theorem and ultradistributions with support in a submanifold, J. Fac. Sci. Univ. Tokyo Sect. IA Math. 24 (1977), no. 3, 607-628. MR 0477770

[13] Hikosaburo Komatsu, Ultradistributions. III. Vector-valued ultradistributions and the theory of kernels, J. Fac. Sci. Univ. Tokyo Sect. IA Math. 29 (1982), no. 3, 653-717. MR687595

[14] Stevan Pilipović and Bojan Prangoski, On the convolution of Roumieu ultradistributions through the $\epsilon$ tensor product, Monatsh. Math. 173 (2014), no. 1, 83-105, DOI 10.1007/s00605013-0503-4. MR.3148662

[15] S. Pilipović and D. Scarpalezos, Regularity properties of distributions and ultradistributions, Proc. Amer. Math. Soc. 129 (2001), no. 12, 3531-3537, DOI 10.1090/S0002-9939-01-06013-0. MR:1860484

[16] Luigi Rodino, Linear partial differential operators in Gevrey spaces, World Scientific Publishing Co., Inc., River Edge, NJ, 1993. MR1249275

[17] Charles Roumieu, Ultra-distributions définies sur $R^{n}$ et sur certaines classes de variétés différentiables (French), J. Analyse Math. 10 (1962/1963), 153-192, DOI 10.1007/BF02790307. MR0158261

[18] Walter Rudin, Real and complex analysis, 2nd ed., McGraw-Hill Book Co., New YorkDüsseldorf-Johannesburg, 1974. McGraw-Hill Series in Higher Mathematics. MR0344043

[19] Michael Ruzhansky and Niyaz Tokmagambetov, Nonharmonic analysis of boundary value problems, Int. Math. Res. Not. IMRN 12 (2016), 3548-3615, DOI 10.1093/imrn/rnv243. MR.3544614

[20] Michael Ruzhansky and Niyaz Tokmagambetov, Very weak solutions of wave equation for Landau Hamiltonian with irregular electromagnetic field, Lett. Math. Phys. 107 (2017), no. 4, 591-618, DOI 10.1007/s11005-016-0919-6. MR3623273

[21] M. Ruzhansky and N. Tokmagambetov, Nonharmonic analysis of boundary value problems without WZ condition, Math. Model. Nat. Phenom. 12 (2017), no. 1, 115-140, DOI 10.1051/mmnp/201712107. MR3614589

[22] R. T. Seeley, Integro-differential operators on vector bundles, Trans. Amer. Math. Soc. 117 (1965), 167-204, DOI 10.2307/1994203. MR0173174

[23] R. T. Seeley, Eigenfunction expansions of analytic functions, Proc. Amer. Math. Soc. 21 (1969), 734-738, DOI 10.2307/2036458. MR0240835

[24] Yoshiko Taguchi, Fourier coefficients of periodic functions of Gevrey classes and ultradistributions, Yokohama Math. J. 35 (1987), no. 1-2, 51-60. MR928372

[25] Yoshiko Taguchi, The universality of the spaces of ultradistributions $\mathcal{C}_{s}(\mathbf{T})^{\wedge}, \mathcal{C}_{(s)}(\mathbf{T})^{\wedge}$ $(0<s \leq \infty), \mathcal{C}_{0}(\mathbf{T})^{\wedge}$ and $\operatorname{Exp}\left(\mathbf{C}^{\times}\right)^{\wedge}$, Tokyo J. Math. 10 (1987), no. 2, 391-401, DOI 10.3836/tjm/1270134522. MR926251

[26] Đorđe Vučković and Jasson Vindas, Eigenfunction expansions of ultradifferentiable functions and ultradistributions in $\mathbb{R}^{n}$, J. Pseudo-Differ. Oper. Appl. 7 (2016), no. 4, 519-531, DOI 10.1007/s11868-016-0157-9. MR 3562483 
École polytechnique fédérale de Lausanne, Faculté des Sciences, CH-1015 Lausanne, SWITZERLAND

Current address: Department of Mathematics, Imperial College London, 180 Queen's Gate, London SW7 2AZ, United Kingdom

Email address: aparajita.dasgupta@epfl.ch

Department of Mathematics, Imperial College London, 180 Queen's Gate, London SW7 2AZ, United KingDOM

Email address: m.ruzhansky@imperial.ac.uk 